\title{
sciendo
}

Current Issues in Pharmacy and Medical Sciences

Formerly ANNALES UNIVERSITATIS MARIAE CURIE-SKIODOWSKA, SECTIO DDD, PHARMACIA

\section{The effect of Ketogenic diet on vitamin D3 and testosterone hormone in patients with diabetes mellitus type 2}

\author{
Hayder Almsaid ${ }^{*}$, Hydar Muhsin Khalfa ${ }^{\circledR}$
}

Department of Biology, Faculty of Science, University of Kufa, Iraq

\section{ARTICLE INFO \\ Received 11 November 2019 \\ Accepted 15 May 2020}

\section{Keywords:}

Ketogenic diet,

testosterone,

lipid profile.

\begin{abstract}
A keto diet is well-known for being a low carb diet in which the body produces ketones in the liver to be used as energy. When something high in carbs is eaten, the body will produce glucose and insulin. Glucose is the easiest molecule for the body to convert and use as energy, so it will be chosen over any other energy source. The aim of this study is to examine the effect of a ketogenic diet on type 2 diabetic patients and the effect it has on testosterone, vitamin D3, HDL, LDL levels, in comparison to non-ketogenic diet subjects. In the study, Type 2 diabetic patients undergoing a keto diet were selected and serum D3 levels and testosterone levels were examined and compared with control subjects. The result show a significant increase in testosterone hormone in patients with diabetes mellitus type 2 following a Ketogenic diet (mean \pm Std. Error 427.4 \pm 2.52 ) as compared with the control group (mean \pm Std. Error $422.2 \pm 0.24$ ) and as compared with patients with diabetes mellitus type 2 who are not following a Ketogenic diet (mean \pm Std. Error $151.4 \pm 1.41$ ). The results show no significant level in LDL level in patients with diabetes mellitus type 2 following a Ketogenic diet (mean \pm Std. Error 78.53 \pm 0.17 ), as compared to a control group (mean \pm Std. Error 75.0.3 \pm 0.14 ) and no significant level in HDL level in patients with diabetes mellitus type 2 following a Ketogenic diet (mean \pm Std. Error $46.3 \pm 1.55$ ), as compared with a control group (mean \pm Std. Error 46.2 \pm 2.43 ), and with patients with diabetes mellitus type 2 who are not following a Ketogenic diet (mean \pm Std. Error $45.1 \pm 1.55)$. The results show a significant increase in vitamin D3 level in patient with diabetes mellitus type 2 who are following a Ketogenic diet (mean \pm Std. Error 53.5 \pm 0.32 ), as compared with a control group (mean \pm Std. Error $57 \pm 0.24$ ), and with patients with diabetes mellitus type 2 who are not following a Ketogenic diet (mean \pm Std. Error 25.1 \pm 1.55 ). Herein, normal vitamin D3 levels in patients corresponds to normal testosterone hormone levels. In conclusion, this study shows that in patients with diabetes mellitus type 2, following a ketogenic diet has a positive effect on the patients' health.
\end{abstract}

\section{INTRODUCTION}

When someone eats something that has high carbohydrates content, the body will produce insulin and glucose. Glucose is the easiest molecule for the body to convert and use as energy, so it will be chosen over any other energy source [1]. Insulin is produced to process the glucose in the bloodstream. Since glucose is being used as a primary energy, fats are not needed and are therefore stored [2]. Typically on a normal, higher carbohydrate diet, the body will use glucose as the main form of energy. By lowering the intake of carbs [3], less fat is stored.

Ketosis is an everyday process within the body, regardless of the amount of carbs consumed, The body can adapt very

\footnotetext{
* Corresponding author

e-mail: haiderl.ligan@uokufa.edu.iq
}

well to processing different types of nutrients into the fuels that it needs. Proteins, fats, and carbs can all be processed for use [4]. Eating a low carb, high fat diet just ramps up this process, which is a normal and safe chemical reaction [5]. When carbohydrate-based foods or excess amounts of protein are consumed, the body will break this down into glucose. Glucose is needed in the creation of ATP, which is a fuel that is needed for daily activity and bodily maintenance [6].

Vitamin D plays an important role in the metabolism of calcium and phosphorus ions, Its primary activities involve absorption of intestinal calcium and reabsorption of renal calcium, and, additionally, it has an immediate impact on chondrocyte and osteoblast differentiation - resulting in 
bone formation [7]. Vitamin D enters the body as two sorts, as D2 or (ergocalciferol) and as D3 or (cholecalciferol). Both D2 and D3 can be produced under ultraviolet B irradiation (UV light). D2 is synthesized in plants from ergosterol, however, D3 is generated by the cells of the epidermis from 7-dehydrocholesterol, after the subtraction of ring B from the molecules of cholesterol. D3 is additionally obtained from nutritional sources [8]. From accumulating evidence from human studies, vitamin $\mathrm{D}$, aside from its regulatory effects on musculoskeletal health, plays a role in reproduction in both sexes [9]. The basis of the exchange between vitamin $\mathrm{D}$ and reproduction lays on the existence of both vitamin D receptors (VDR) and $1 \alpha$-hydroxylase (CYP27B1) enzyme in the reproductive organs [10]. In the males, VDR are existent in the testis, epididymis, prostate, and seminal vesicles [11]. In Sertoli cells, as ion channel dependent secretory activity, vitamin D appears to stimulate calcium intake through activitation of the nuclear receptors [12].

Testosterone is the main steroid hormone of sex in the male and is synthesized by transformation of androstenedione through $17 \beta$-hydroxysteroid dehydrogenase. This hormone will then be converted by $5 \alpha$-reductase into dihydrotestosterone [13]. Testosterone is secreted by the leydig cells under the influence of $\mathrm{LH}$, and acts as negative feedback factor on the hypothalamus, to decrease the release of GnRH. Furthermore, it acts specifically on the anterior pituitary gland, therefore, inhibiting the release of LH hormone [14]. Testosterone is the fundamental steroid hormone for spermatogenesis and for developing secondary sexual characteristics [15]. The influences of Testosterone are various, and wide; it acts on stimulating the development of bone connective tissue and muscle cells, and on skin, it acts to increase the size and secretion of the sebaceous gland, and to stimulate development and growth of testicles, prostate gland, seminal vesicles and penis. Testosterone has plays a fundamental role in spermatogenesis [16].

\section{MATERIALS AND METHODS}

\section{Subjects}

Subjects were selected from the Endocrinology Department at the Al-sader Teaching Hospital in Alnajaf Province, Iraq.

Serum specimen was collected from male patients with diabetes mellitus type 2 who are following the Ketogenic diet (low carbohydrates diet) $(n=30)$, patients with diabetes mellitus type 2 who are not following the Ketogenic diet $(n=30)$ and normal patients (patients with non-Ketogenic diet as a control) $(n=30)$. Participants for this study had an age range between 30-41 years. The total samples tested are 90 samples. All subjects included in this study were consenting patients who gave both written and verbal approval.

\section{ELISA}

In the 90 samples, Vitamin D3 and testosterone Levels were measured by utilizing the immunological method (Enzyme-Linked-Imuno-Sorbent Assay) by applying an ELISA reader (Huma Germany origin). All specimens and reagents were allowed to come to room temperature before use. All reagents were mixed softly without foaming. Once the procedure has started, all steps were completed without interruption. All biochemical tests were conducted in the laboratories of the Biology Department/faculty of Sciences/ University of Kufa. The ELISA kits used in this study was testosterone (ab108666), vitamin D3 (ab213966) and Cholesterol Assay Kit - HDL and LDL (ab65390) - abcam Company USA in Origin.

\section{RESULTS AND DISCUSSION}

The result show a significant increase in testosterone hormone in patients with diabetes mellitus type 2 following the Ketogenic diet (mean \pm Std. Error 427.4 \pm 2.52 ), as compared with the control group (mean \pm Std. Error $422.2 \pm 0.24$ ), and as compared with patients with diabetes mellitus type 2 who are not following the Ketogenic diet (mean \pm Std. Error 151.4 \pm 1.41 ). Herein, it appears that the cholesterol intake of those following the control is not enough to increase testosterone and it would appear that it is necessary to ingest an amount of cholesterol compatible to that of the Ketogenic diet to increase testosterone [17]. The results also show no significant level in LDL level changes in patients with diabetes mellitus type 2 following the Ketogenic diet (mean \pm Std. Error 78.53 \pm 0.17 ), in comparison with the control group (mean \pm Std. Error $75.0 .3 \pm 0.14$ ) and with patients with diabetes mellitus type 2 who are not following the Ketogenic diet (mean \pm Std. Error $75.8 \pm 0.44)$. Moreover, the results show no significant level in HDL levels in patients with diabetes mellitus type 2 who are following the Ketogenic diet (mean \pm Std. Error $46.3 \pm 1.55$ ), as compared with the control group (mean \pm Std. Error 46.2 \pm 2.43 ) and as compared with patients with diabetes mellitus type 2 who are not following the Ketogenic diet (mean \pm Std. Error 45.1 \pm 1.55 ). This result is probably attributable to loss of body fat and carbohydrate restriction. However, while the Ketogenic diet is normocaloric, it does not appear to alter the lipid profile to any significant degree, since lipid levels did not exceed the reference range [18].

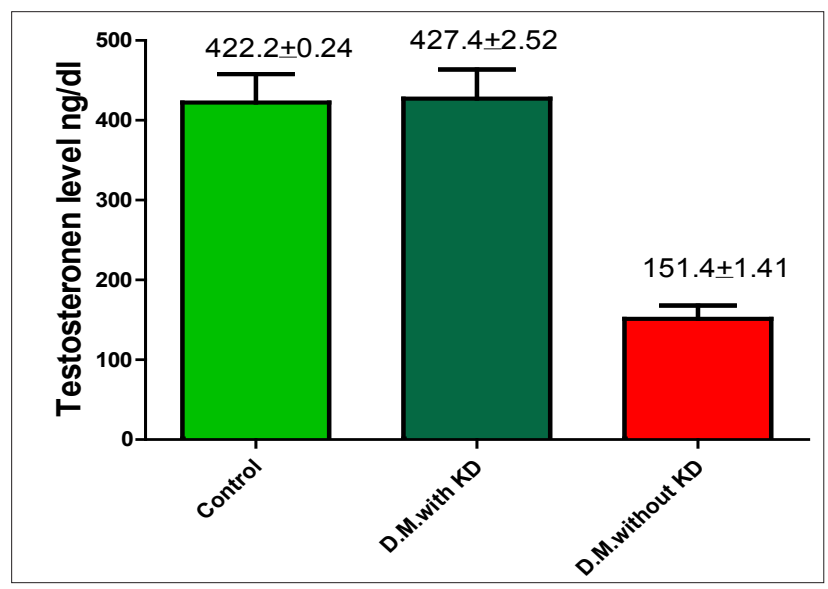

Note the significant differences $(p<0.05)$

Figure 1. The comparison of Testosterone level in the serum between control Group and patients with diabetes mellitus type 2 who are following the Ketogenic diet (D.M. with KD) and patients with diabetes mellitus type 2 who are not following the Ketogenic diet (D.M. without KD) 


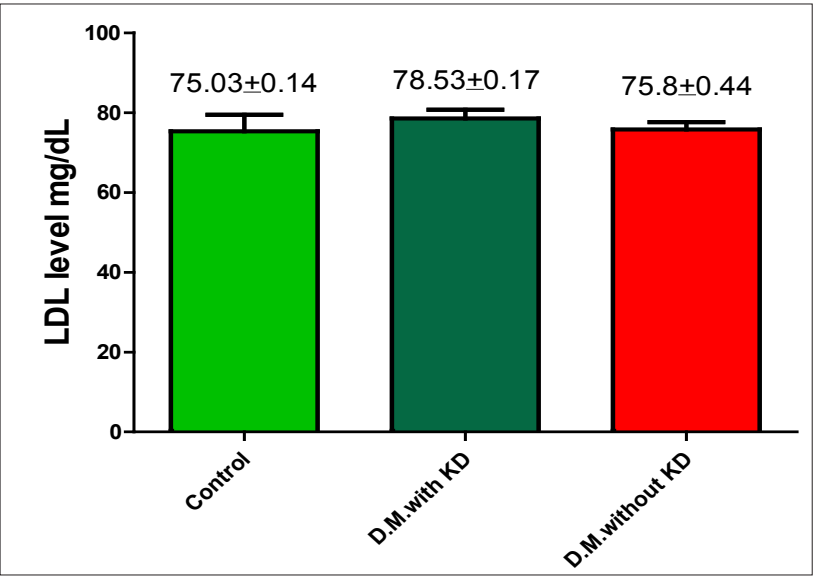

Note that there is no significant difference $(p<0.05)$

Figure 2. The comparison of LDL level in the serum between control Group and patients with diabetes mellitus type 2 who are following the Ketogenic diet (D.M. with KD) and patients with diabetes mellitus type 2 who are not following the Ketogenic diet (D.M. without KD)

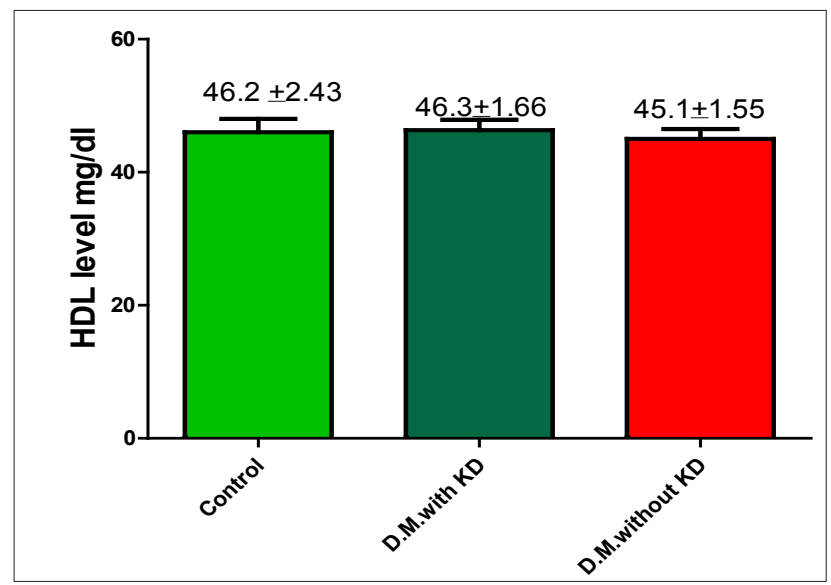

Note that there is no significant differences $(p<0.05)$

Figure 3. A comparison of HDL level in the serum between control Group, patients with diabetes mellitus type 2 who are following the Ketogenic diet (D.M. with KD) and patients with diabetes mellitus type 2 who are not following the Ketogenic diet (D.M. without KD)

The results show a significant increase in vitamin D3 level in patients with diabetes mellitus type 2 who are following the Ketogenic diet (mean \pm Std. Error 53.5 \pm 0.32 ), in comparison with the control group (mean \pm Std. Error $57 \pm 0.24$ ) and with patients with diabetes mellitus type 2 who are not following the Ketogenic diet (mean \pm Std. Error $25.1 \pm 1.55)$. Herein, probably for each kilogram of weight loss, vitamin D concentration increased. These findings are in contrast with previously published literature that suggested a negative effect of Ketogenic diet on bone composition, because ketoacidosis generated by low-carbohydrate/ high-protein diets results in hypercalciuria [19].

Overall, there is a clear and precise relationship between patients undergoing a ketogenic diet and those with a normal diet. Blood parameters such as vitamin D3 levels appear to increase in patients following the ketogenic diet and who are type 2 diabetics. This shows a positive relationship in that a low carbohydrates diet in type 2 diabetics results in elevated vitamin D3 levels. Testosterone levels also increased in ketogenic diet diabetic patients. This can explain the presence of a high protein and fats within the body leading to increased levels of energy and muscle build up.

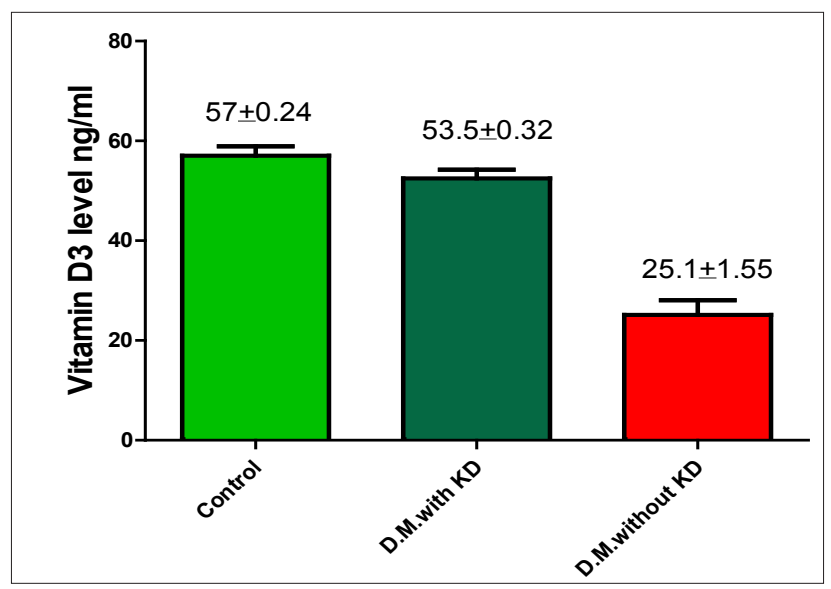

Note the significant differences $(p<0.05)$

Figure 4. A comparison of vitamin D3 level in the serum between control Group, patients with diabetes mellitus type 2 following the Ketogenic diet (D.M. with KD) and patients with diabetes mellitus type 2 who are not following the Ketogenic diet (D.M. without $\mathrm{KD}$ )

\section{CONCLUSIONS}

From the result of this study, in patients with diabetes mellitus type 2, the type of diet followed can have positive effects on health vitamin D3 and testosterone levels. The increase and normalization of these levels can be attributed to high levels of protein in the subjects' diet, and the lack of carbohydrates. In general, a ketogenic diet can effect vitamin D3 and testosterone levels in patients with type 2 diabetes mellitus as compared to control subjects.

\section{ORCID iDs}

Hayder Almsaid (Dhttps://orcid.org/0000-0001-8981-4884 Hydar Muhsin Khalfa (Dhttps://orcid.org/0000-0003-0665-5174

\section{REFERENCE}

1. Hamada T, Hayashi T, Kimura T, Nakao K, Moritani T. Electrical stimulation of human lower extremities enhances energy consumption, carbohydrate oxidation, and whole body glucose uptake. J App Physiol. 2004:911-6.

2. Fabbrini E, Faidon M, Mohammed BS, Pietka T, Abumrad NA, Patterson BW, Okunade A, Klein S. Intrahepatic fat, not visceral fat, is linked with metabolic complications of obesity. PNAS. 2009;106(36):15430-5.

3. Chu S, Majumdar A. Opportunities and challenges for a sustainable energy future. Nature. 2012;488(7411):294-303.

4. Paoli A. Ketogenic diet for obesity: friend or foe? IJERPH. 2014; 11(2):2092-107.

5. Bozzetto L, Prinster A, Annuzzi G, Costagliola, L, Mangione A, Vitelli A, et al. Liver fat is reduced by an isoenergetic MUFA diet in a controlled randomized study in type 2 diabetic patients. Diabetes care. 2012;35(7):1429-35.

6. Leist BM, Single B, Castoldi AF, Kühnle S. A Switch in the decision between apoptosis and necrosis. J Exp Med. 1997;185(8):1481-6.

7. DiMeglio LA, Imel EA. Calcium and phosphate: hormonal regulation and metabolism. In Basic and applied bone biology. Academic Press. 2019:257-82. 
8. Pritchard L, Lewis S, Hickson M. Comparative effectiveness of vitamin D supplementation via buccal spray versus oral supplements on serum systematic review protocol. JBI Evidence Synthesis. 2019;17(4):487-99.

9. Marquina C, Mousa A, Scragg R, De Courten B. Vitamin D and cardiometabolic disorders : a review of current evidence, genetic determinants and pathomechanisms. Obesity Rev. 2019;20(2):262-77.

10. Jueraitetibaike K, Zheng D, Dan-Dan W, Long-Ping P, Jun J, Li C, et al. The effect of vitamin D on sperm motility and the underlying mechanism. AJA. 2019;21(4):400.

11. Cito G, Cocci A, Micelli E, Gabutti A, Russo GI, Coccia ME, et al. Vitamin D and male fertility: an updated review. World J Men's Health. 2020;38(2):164-77.

12. Harchegani AB, Irandoost A, Mirnamniha M, Rahmani H, Tahmasbpour E, Shahriary A. Possible mechanisms for the fffects of calcium deficiency on male infertility. Int J Fertil Steril. 2019; 12(4):267.

13. Tang R, Shen Y, Wang M, Zhou H, Zhao Y, Wang M. Highly efficient synthesis of boldenone from androst-4-ene- 3, 17-dione by Arthrobacter simplex and Pichia pastoris ordered biotransformation. Bioproc Biosyst Eng. 2019;42(6):933-40.
14. Schulz RW, Taranger GL, Bogerd J, Nijenhuis W, Norberg B, Male R, Andersson E. Entry into puberty is reflected in changes in hormone production but not in testicular receptor expression in Atlantic salmon (Salmo salar). Reprod Biol Endocrinol. 2019;17(1):48.

15. Chung JY, Brown S, Chen H, Liu J, Papadopoulos V, Zirkin B. Effects of pharmacologically induced leydig cell testosterone production on intratesticular testosterone and spermatogenesis Jin-Yong Chung. BOR. 2020;102(2):489-98.

16. Article R. Biological and psychological influences of cross-sex hormone in transgender. Med Health. 2019;14(1):1-22.

17. Santos HO. Ketogenic diet and testosterone increase: Is the increased cholesterol intake responsible? To what extent and under what circumstances can there be benefits. IJEM. 2017;16(3):266-70.

18. Dashti HM, Mathew TC, Khadada M, Al-Mousawi M, Talib H, Asfar SK. Beneficial effects of ketogenic diet in obese diabetic subjects. Mol Cell Biochem. 2007;302(1-2):249-56.

19. Huynh T, Rm G, Nyunt O, Bowling F, Cowley D, Gm L. The association between ketoacidosis and $25(\mathrm{OH})$ - vitamin D 3 levels at presentation in children with type 1 diabetes mellitus. Pediat Diabetes. 2009;10(1):38-43. 